\title{
È possibile una gestione "ideale" della pandemia da malattia renale cronica?
}

\author{
Stefano Michelassi \\ SOC Nefrologia e Dialisi, Ospedale S.M. Annunziata - Firenze 2, USL Toscana Centro, Firenze - Italy
}

\begin{abstract}
Is there an "ideal" management of Chronic Kidney Disease?
Chronic Kidney Disease (CKD) is recognized as one of the major categories of noncommunicable epidemic diseases and in the last decades it has been largely growing in incidence and prevalence all over the world. Ideal management of CKD pandemic should be comprehensive of measures of tertiary, secondary, primary and primordial prevention. So, it should include prompt diagnosis and treatment of traditional and non-traditional risk factors for CKD, optimal conservative treatment for non-dialysis dependent CKD patients and appropriated dialysis therapy or renal transplantation for patients with end-stage renal disease. However, these goals are not easy to obtain on a global scale. It would be possible only by a broad and holistic approach, ranging from good governance to achievement of the sustainable development goals (SDGs).
\end{abstract}

Keywords: CKD prevention, food deserts, PM2.5, SDGs

\section{Analisi quantitativa del problema}

La malattia renale cronica (MRC) è oggi considerata una malattia epidemica non trasmissibile alla stregua di altre malattie tradizionalmente tenute in più alta considerazione dall'opinione pubblica (e purtroppo anche da parte della comunità scientifica) come il diabete, le malattie cardiovascolari, le malattie polmonari croniche e le neoplasie.

Anche per differenze fra i vari studi riguardo al tipo di popolazione studiata, la prevalenza della MRC nell'adulto è varialmente stimata dal 7\% in alcuni studi italiani (GUBBIO, CARHES) fino al $15 \%$ nell'ultimo report annuale USRDS (1).

Per i dati sulla MRC in tutte le età possiamo riferirci invece ai risultati del Global Burden of Disease (GBD) (2), un vasto programma mondiale (coinvolti oltre 1.800 ricercatori in 127 Paesi) di ricerca che valuta la mortalità e la disabilità causate dalle più importanti malattie e condizioni di rischio. Proprio da questi dati emerge l'importanza della pandemia da MRC, sia

Received: November 2, 2021

Accepted: November 3, 2021

Published online: January 8, 2022

Indirizzo per la corrispondenza:

Stefano Michelassi

SOC Nefrologia e Dialisi

Ospedale S.M. Annunziata - Firenze 2

USL Toscana Centro

Firenze - Italy

stefano.michelassi@uslcentro.toscana.it in termini assoluti di prevalenza e di incidenza, dato che nel 2016 risultavano affetti da MRC in tutto il globo 276.000 .000 di individui di cui 21.000 .000 avevano ricevuto la diagnosi in quell'anno, sia soprattutto in termini di andamento temporale. Nei 25 anni precedenti, dal 1990 al 2016, incidenza (da 215 a 289 pazienti/100.000 abitanti) e prevalenza (da 2.804 a 3.732 pazienti/100.000 abitanti) erano aumentate entrambe del $25 \%$ e, per il contemporaneo aumento della popolazione, a livello globale l'aumento era rispettivamente dell' $89 \%$ e dell' $87 \%$ ! In modo simile, nello stesso periodo di tempo, mortalità (da 11 a 16 decessi/100.000 abitanti) e giorni di buona salute persi (DALYs, da 2.803 a 3.733 ) a causa della MRC erano aumentati entrambi del $29 \%$ e, a livello globale, rispettivamente del $98 \%$ e dell' $87 \%$. Inoltre, dal momento che esiste inevitabilmente un "sottobosco" di casi non diagnosticati, i dati reali sono sicuramente peggiori, tanto che alcuni Autori ipotizzano che fra i 5 e i 10 milioni di decessi nel mondo ogni anno siano attribuibili alla MRC, in misura simile alla mortalità per neoplasie, diabete e malattie respiratorie.

La MRC non interessa però il nostro pianeta in modo omogeneo; viceversa, esistono significative differenze regionali, in particolare a seconda delle condizioni socio-economiche dei vari Paesi. Una volta standardizzata rispetto all'età della popolazione, la prevalenza della MRC appare sproporzionatamente elevata nei Paesi economicamente più svantaggiati e a più basso indice socio-demografico (ISD) (Figg. 1 e 2). In questi ultimi essa non solo è più frequente ma compare anche più spesso in individui di età meno avanzata, adolescenti e giovani adulti. 


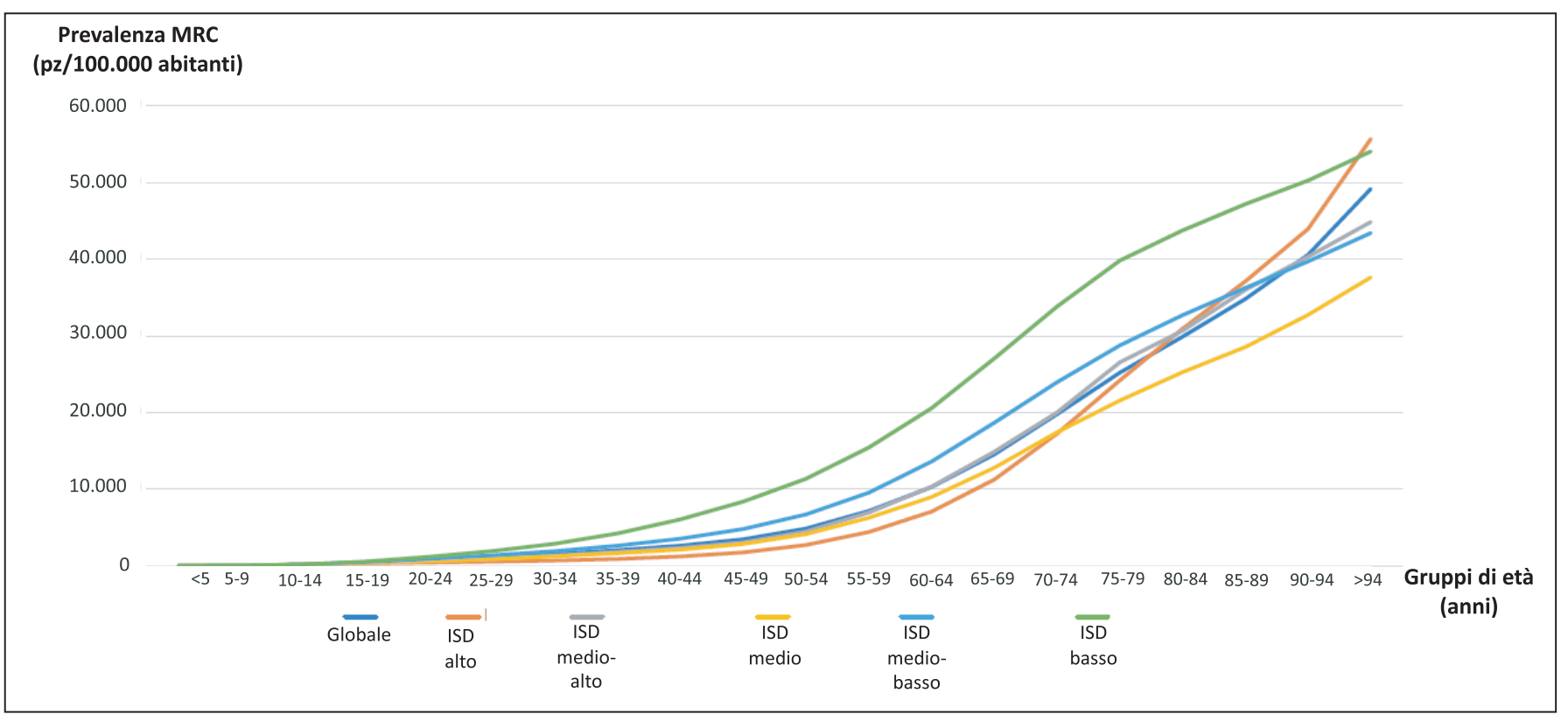

Fig. 1 - Prevalenza della MRC per età e ISD. Da (2) modificato.

\section{INDICE SOCIO-DEMOGRAFICO (ISD)}

Sintetizza in un numero il livello di sviluppo socio-demografico di un territorio.

E' una media composita di valori che analizzano il reddito pro-capite, il livello scolastico medio e la fertilità totale

Varia da 0 a 1 :

$0=$ minor reddito, più basso livello scolastico, più alta fertilità

1 = maggior reddito, più alto livello scolastico, più bassa fertilità

Fig. 2 - Indice Socio-Demografico (ISD).

\section{Analisi delle cause e dei fattori di rischio di MRC}

Una gestione ottimale della MRC dovrebbe comprendere misure di prevenzione primaria (cioè il trattamento dei suoi fattori di rischio), secondaria (cioè rallentarne la velocità di progressione) e terziaria (intendendo con tale termine l'ottimizzazione del trattamento sostitutivo per i pazienti che ne siano giunti allo stadio terminale).

Il primo punto è, quindi, comprendere quali siano i principali fattori di rischio.

I fattori di rischio per MRC possono essere inizialmente divisi in due grandi categorie, fattori epidemiologici, relativi alla popolazione, e fattori di rischio pr.d. (propriamente detti), relativi al singolo individuo.

Fattori epidemiologici: i principali fattori epidemiologici responsabili nel mondo dell'aumento dei DALYs dovuti a MRC dal 1990 al 2016 sono stati individuati nella struttura di età delle popolazioni (dato "trasversale") e nel loro andamento di crescita nel tempo (dato "longitudinale") (Fig. 3). Ma c'è una sostanziale differenza tra questi due fattori. II contributo

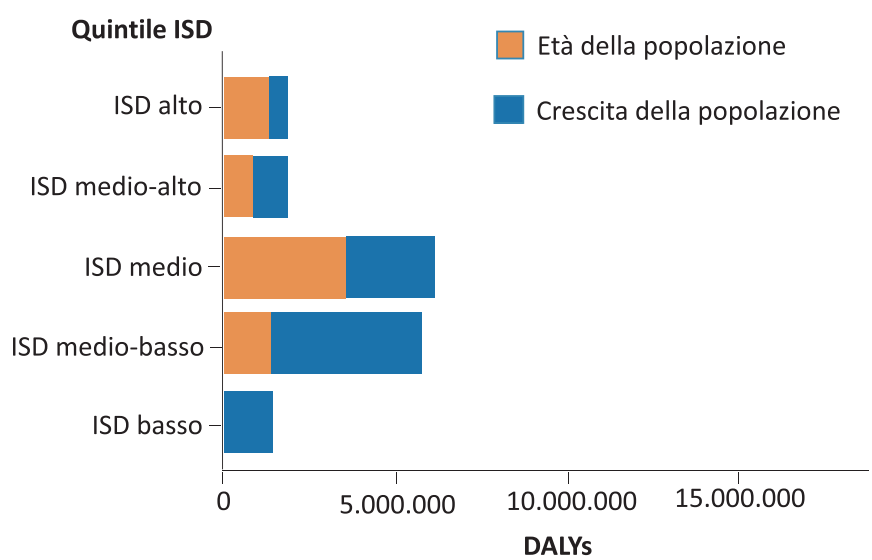

Fig. 3 - Variazioni degli anni di vita persi o vissuti con disabilità per MRC (DALYs) dal 1990 al 2016 dipendenti da fattori epidemiologici relativi alla popolazione a seconda dell'ISD. Da (2) modificato.

dell'invecchiamento è prevalente nei Paesi ad alto ISD, perché gli individui vivono più a lungo e quindi hanno maggiori probabilità di sviluppare la malattia. Invece, il contributo della crescita della popolazione è prevalente nei Paesi a ISD basso, caratterizzati dal rapido aumento del numero di individui che vivono in condizioni di scarse risorse igienico-sanitarie e quindi a maggior rischio di MRC.

Fattori di rischio pr.d.: i principali fattori di rischio per la $M R C$, universalmente riconosciuti, sono diabete, ipertensione arteriosa e glomerulonefriti. Questi medesimi fattori sono anche quelli responsabili dell'aumento dei DALYs dovuti a MRC dal 1990 al 2016 (Fig. 4). In questo caso, però, i contributi del diabete e dell'ipertensione crescono proporzionalmente all'aumentare dell'ISD, mentre il contributo delle glomerulonefriti ha un andamento opposto. 


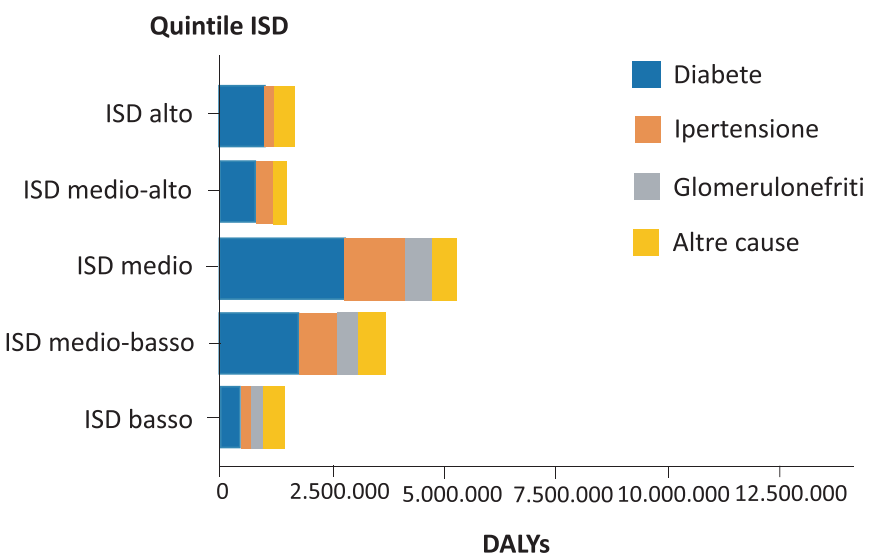

Fig. 4 - Variazioni degli anni di vita persi o vissuti con disabilità per MRC (DALYs) dal 1990 al 2016 dipendenti dalle 4 cause principali di MRC a seconda dell'ISD. Da (2) modificato.

\section{La gestione ideale della MRC}

II notevole impatto dell'ipertensione e del diabete sulla MRC, in particolare nei Paesi a medio-alto ISD, porta a conseguenze significative anche sulle modalità ottimali di screening per MRC in queste popolazioni. Una revisione sistematica di vari studi (3) ha concluso che lo screening per $M R C$, mediante dosaggio della proteinuria e/o del filtrato glomerulare stimato (eGFR), dovrebbe essere effettuato selettivamente nelle sottopopolazioni ad alto rischio, cioè ipertesi, diabetici e anziani, e nei gruppi etnici a maggior rischio. Analizzare tutta la popolazione si rivelerebbe una strategia certamente più efficace ma non efficiente, perché $i$ costi supererebbero i benefici.

Appare quindi razionale pensare a un percorso condiviso fra MMG e nefrologo per la gestione ottimale della MRC, come proposto da diverse autorità scientifiche $(4,5)$. II MMG ha il compito di individuare i pazienti a rischio (principalmente anziani, ipertesi e diabetici), indagarli per malattia renale ed inviare quelli nefropatici o a maggior rischio al nefrologo. Quest'ultimo valuterà, caso per caso, la necessità di prendere in carico il paziente inviato o, viceversa, di reindirizzarlo al MMG con i consigli opportuni per il proseguimento del follow-up (Fig. 5). Infine, è implicito che i pazienti cronici correttamente diagnosticati siano trattati in modo ottimale dal nefrologo con terapia conservativa $\mathrm{o}$, in fase terminale, con qualsiasi forma di trattamento sostitutivo. Tuttavia tutte queste tappe, semplici sulla carta, trovano grossi ostacoli nel mondo reale.

\section{La gestione reale della MRC}

Dal momento che il percorso di gestione della MRC prevede, come compiti fondamentali, prima la diagnosi (possibilmente precoce) dei fattori di rischio e successivamente quella della malattia renale, se questi non vengono assolti cade tutto il resto. Per quanto riguarda il diabete, si stima che i casi non diagnosticati oscillino tra il $27 \%$ nelle Americhe e il $63 \%$ nell'Africa subsahariana e che in Italia arrivino al $36 \%(6)$. In tutto il mondo, la diagnosi di ipertensione arteriosa sfugge nel $50 \%$ dei casi (7). Altrettanto precaria, anche nei Paesi più sviluppati, è la situazione riguardo alla diagnosi di MRC. Per esempio si stima che negli USA solo il $12 \%$ dei pazienti totali affetti da MRC sia consapevole della malattia e questa percentuale aumenta "solamente" fino al $62 \%$ per i pazienti allo stadio 4 e all' $86 \%$ per quelli allo stadio 5 (1).

Ma quelli diagnostici non sono i soli problemi. Difficoltà significative nella gestione della MRC nel mondo reale possono esistere anche per i pazienti in cui la malattia sia stata correttamente riconosciuta, in questo caso essenzialmente rappresentate da una difficoltà di accesso alle cure che trova le sue radici in due cause non mutuamente esclusive:

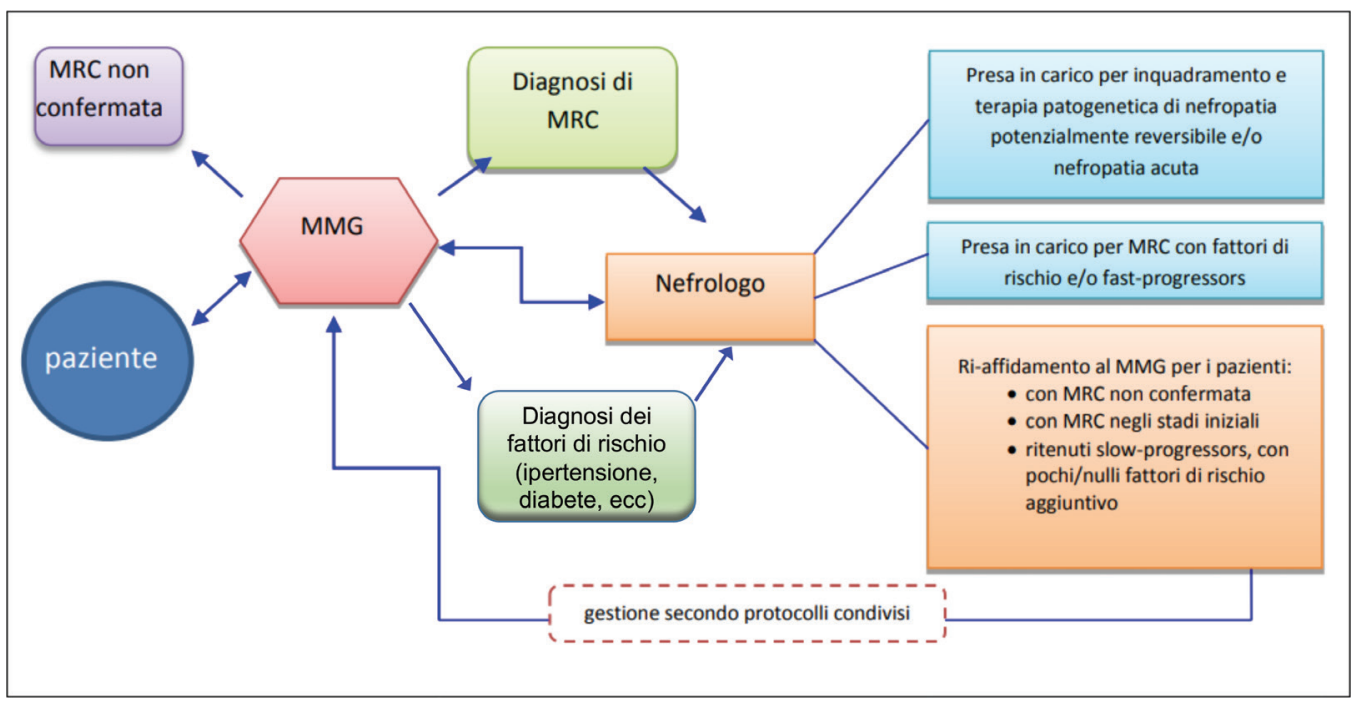

Fig. 5 - Percorso condiviso fra MMG e nefrologo per la gestione ottimale della MRC secondo il documento di indirizzo del Ministero della Salute italiano (5 novembre 2014). Da (4) modificato. 
- in molti Paesi la difficile accessibilità alle cure è dovuta a una carenza di risorse umane e tecnologiche. La stessa disponibilità di nefrologi varia ampiamente da Paese a Paese. In un lavoro apparso sull'American Journal of Kidney Diseases nel 2014 (8) la prevalenza di nefrologi in 17 Paesi europei variava da 5,3 per 100.000 abitanti in Italia a 0,5 per 100.000 abitanti in Irlanda. Nei Paesi in via di sviluppo questi numeri scendono ulteriormente in modo drammatico (9), basti pensare che nell'Africa subsahariana la maggior parte dei Paesi ha meno di 10 nefrologi! Inoltre, ai fini di una gestione ottimale della pandemia di MRC può risultare critica anche la disponibilità di risorse "tecnologiche" in senso lato. Prendendo in esame i Paesi a basso reddito, a un livello di cure primario, nel $41 \%$ dei casi non è possibile dosare la creatinina sierica, nell' $82 \%$ non è possibile avere un dosaggio quantitativo dell'albuminuria e nel $18 \%$ neanche un semplice esame delle urine. Negli stessi Paesi, a livello secondario/terziario di cure non esiste la possibilità di misurare l'albumina urinaria nel $35 \%$ dei casi, nel $18 \%$ è rara la disponibilità di un'ecografia addominale e la biopsia renale non è possibile nel $53 \%$ dei casi. La situazione migliora, ovviamente, in modo progressivo passando ai Paesi a fascia di reddito via via più alta (9);

- in alcuni casi, tuttavia, l'accesso alle cure è difficoltoso anche quando le risorse sarebbero di per sé disponibili. In questi casi il principale fattore causale è costituito dalla tipologia dei sistemi di finanziamento della MRC nei suoi aspetti diagnostici e terapeutici. Nel mondo la gestione della MRC in terapia conservativa è principalmente finanziata da un sistema misto pubblico e privato, ma, mentre nei Paesi a più alto reddito ha un finanziamento prevalentemente pubblico e gratuito, in quelli a reddito più basso è più spesso affidata a risorse esclusivamente private. II problema diventa enorme quando andiamo a considerare la gestione della MRC in terapia sostitutiva (rappresentata per la stragrande maggioranza dalla dialisi), molto più costosa. Nella maggior parte dei Paesi questa è completamente gratuita ma in quelli a medio-basso e basso reddito è più frequente la richiesta di un contributo da parte dei pazienti di grado variabile fino all'accollamento totale del costo. Il problema è accentuato dal fatto che la maggior parte delle persone nei Paesi in via di sviluppo non ha accesso a sistemi di assicurazione sanitaria, il che rende il trattamento dialitico praticamente non utilizzabile (10).

L'effetto combinato di una carenza di risorse associata al costo del trattamento sostitutivo è che in tutto il mondo solo il 7\% dei pazienti trattati appartiene a Paesi in fascia di reddito medio-bassa e bassa contro il 93\% di quelli residenti in Paesi a fascia alta e medio-alta. Per esempio, è stato stimato che nel 2010 in tutto il pianeta, fra i 2.284 .000 e i 7.083.000 pazienti che richiedevano una terapia sostitutiva non la ricevettero. E naturalmente la percentuale stimata di "non trattati", già significativa anche nei Paesi ad alto reddito, aumentava rapidamente fino a valori superiori al $95 \%$ in quelli a basso reddito (11)! In altri termini, in tutto il mondo un numero enorme di pazienti non ha la possibilità di accedere al trattamento sostitutivo (dialisi e trapianto) e ciò si traduce in un numero enorme di "morti evitabili". Anche quando, invece del semplice accesso alle cure, si vanno a utilizzare indici più complessi che combinano I'“accessibilità" alle cure con la "qualità" delle cure, il risultato, ovvio, è che la possibilità di essere curati bene aumenta progressivamente andando dai Paesi più poveri a quelli più ricchi (2).

\section{II problema dei fattori di rischio "non tradizionali"}

Un altro ostacolo a una gestione ottimale della pandemia da MRC sta nel fatto che, oltre ai fattori di rischio tradizionali sopracitati, sono ormai riconosciuti da diversi anni altri fattori di rischio, non tradizionali (Fig. 6). Alcuni di questi, come ad esempio, infezioni urinarie, urolitiasi e sostanze nefrotossiche, sono ben noti e facilmente affrontabili, ma altri lo sono molto meno e il loro trattamento è molto più complicato perché richiederebbe interventi non solo di tipo medico specialistico ma anche a livello di salute pubblica e sociale. Di seguito ne saranno brevemente ricordati alcuni.

\section{FATTORI DI RISCHIO “NON TRADIZIONALI" DI MRC}

- Fumo

- Fattori nutrizionali

- Fattori gestazionali ed embriologici

- Alterazioni climatiche

- Contaminazione da polveri

- Sostanze nefrotossiche

- Urolitiasi

- Infezioni

- Episodi di insufficienza renale acuta

Fig. 6 - Fattori di rischio per MRC non tradizionali.

\section{Fumo}

Il fumo di sigaretta è stato identificato come uno dei più significativi fattori di rischio per la progressione della MRC in alcuni sottogruppi di pazienti (per esempio, nei diabetici) ma il suo rapporto con la MRC nella popolazione generale è meno chiaro. Tuttavia diversi studi ne suggeriscono un ruolo. In due studi prospettici, uno statunitense (12) e uno giapponese (13), la condizione di fumatore era associata a un aumento significativo del rischio di peggiorare il filtrato glomerulare. In uno studio trasversale norvegese (14) gli Autori 
hanno dimostrato un aumento del rischio di MRC significativo e dose-dipendente per un'esposizione cumulativa $>25$ pacchetti/anno.

\section{Fattori nutrizionali}

Errori nutrizionali possono produrre una serie di effetti negativi sull'omeostasi dell'organismo e avere conseguenze negative sulla funzione renale. Se pensiamo che diabete, ipertensione arteriosa e malattia vascolare aterosclerotica costituiscono fattori di rischio per MRC, appare evidente che una dieta ricca di sale, zuccheri raffinati, grassi saturi e proteine animali sia molto meno salutare per il rene rispetto a una con meno sale e più ricca in zuccheri complessi e proteine vegetali. Ma quali sono i motivi che inducono un paziente ad assumere una dieta più o meno "sana"? A prima vista si potrebbe pensare che ciò dipenda esclusivamente dalla capacità del medico di consigliarla e dalla volontà del paziente di seguirla, invece la questione è molto più complessa. Per esempio, un ruolo fondamentale è rivestito dallo stato socioeconomico (SSE) (Fig. 7). Un basso SSE si associa a un maggiore consumo di cibi nocivi per il metabolismo e per l'apparato cardiovascolare (farine raffinate, grassi aggiunti, bevande zuccherine, ecc.) e a un basso consumo di cibi più salutari (ad esempio, farine integrali, frutta, verdura e pesce) (15). Come mai? Sono in gioco più fattori, spesso interconnessi fra loro, come la disponibilità e il tipo di negozi alimentari vicini alla zona di residenza, la disponibilità di trasporti per giungervi, la vicinanza di ristoranti "fast-food" e anche l'accessibilità a parchi pubblici o comunque ad aree adatte a praticare attività motoria, che contribuisce a diminuire il bilancio calorico netto della dieta e il rischio di obesità. In particolare, il consumo di frutta e verdura diminuisce progressivamente con l'aumentare della distanza dai supermercati e dai negozi dove esse sono disponibili (16). È stato coniato il termine "food deserts" per indicare quelle aree in cui l'accesso ai negozi alimentari con cibi più salutari è particolarmente limitato, forzando gli individui ad acquistare alimenti ipercalorici che tendono a essere più economici e a poter essere conservati più a lungo sugli scaffali degli stessi negozi. Ebbene, i "food deserts" sono soprattutto presenti nelle aree rurali e in quelle più povere (17). Nei dintorni di Melbourne la disponibilità di cibi ipercalorici a basso contenuto nutrizionale e il suo rapporto con la disponibilità di frutta e verdura sono maggiori nei supermercati localizzati nelle aree socio-economicamente più svantaggiate (18). Nella Contea di Baltimora, la disponibilità di negozi alimentari con maggiore disponibilità di cibi più sani è straordinariamente più alta nelle aree abitate prevalentemente da popolazione bianca e con reddito medio più elevato, mentre l'accessibilità a ristoranti fast-food è nettamente maggiore nelle aree con popolazione prevalentemente nera e con un più basso reddito (19). Si potrebbe pensare che, nelle aree economicamente più depresse la minore disponibilità di cibi "sani" sia dovuta al loro maggior costo, eccessivo per i residenti. Non è così. Anche per individui economicamente più svantaggiati un più facile accesso a negozi alimentari con maggiore disponibilità di cibi "sani" si associa a un minore introito di alimenti ipercalorici e a elevato contenuto di sodio e fosforo e a un maggiore consumo di frutta e verdura (20).

\section{Fattori gestazionali ed embriologici}

Un basso peso alla nascita e/o un neonato piccolo per età gestazionale si associano a un aumentato rischio di comparsa

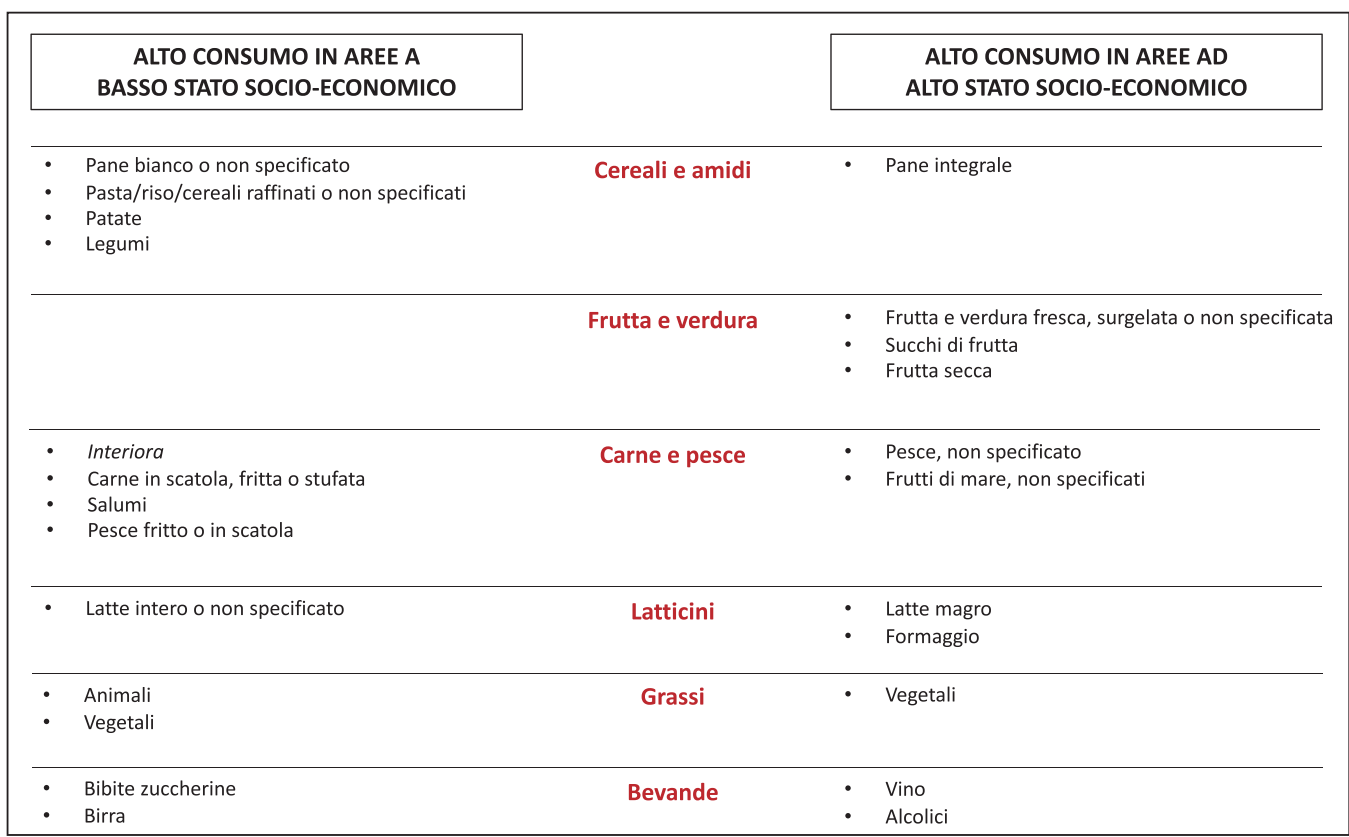

Fig. 7 - Maggior consumo di alimenti in aree a basso e a elevato stato socio-economico. Da (15) modificato. 
di MRC, soprattutto nelle prime decadi di vita ma anche successivamente (21). Alla nascita il numero di nefroni in ciascun rene si aggira mediamente intorno al milione ma in realtà ha un'ampia variabilità interindividuale, a suggerire che esso possa essere il risultato di processi avvenuti durante lo sviluppo intrauterino. Inoltre, popolazioni con minor numero di nefroni (per esempio, gli aborigeni australiani) hanno un'aumentata prevalenza di MRC. Queste osservazioni hanno condotto all'ipotesi che i neonati sottopeso abbiano un minor numero di nefroni alla nascita, che porterebbe, in futuro, a un aumentato rischio di sviluppare una MRC. Nel 2015 la prevalenza di basso peso alla nascita nell'intero globo era stimata al $14,6 \%$ ma anche in questo caso il dato non era uniformemente distribuito perché il $91 \%$ di tutti i nati con basso peso si trovava nei Paesi a basso e medio reddito, principalmente Asia meridionale (48\%) e Africa subsahariana (24\%) (22).

La causa più frequente di basso peso alla nascita, il parto pre-termine o prematuro, si verifica più facilmente (oltre che in caso di età avanzata della madre, pre-eclampsia, ecc.) nelle madri affette da malattie croniche. Per esempio, diabete e obesità materne sono associati a un maggior rischio per il nascituro di sviluppare MRC nel corso della sua vita (23). Ne consegue che prevenire una futura MRC nel nascituro significa avere riguardo per la salute della madre! In questo senso possiamo definire (oltre a quella primaria e secondaria) un terzo tipo di prevenzione "primordiale" che addirittura precede quella primaria, perché effettuata ancora prima che nasca il "paziente" e che si esplica assicurando alle donne (future madri), una vita sana in un ambiente adeguato (dal punto di vista igienico-sanitario nel senso più lato del termine) fin da quando esse sono ancora bambine.

\section{Fattori climatici}

All'inizio degli anni duemila venne descritta per la prima volta una nefropatia cronica endemica dell'America Centrale che colpiva prevalentemente operai che lavoravano all'aperto nei campi di canna da zucchero e che è stata successivamente denominata "nefropatia mesoamericana". La sua patogenesi non è ancora nota con certezza ma il fatto che quei lavoratori mediamente perdessero $2,5 \mathrm{Kg}$ di peso durante una giornata lavorativa nonostante l'assunzione di 5-6 L di acqua suggerisce che la disidratazione o lo stress da calore possano avere un ruolo importante. Oggi noi sappiamo che esistono anche altre nefropatie endemiche da causa non nota (in Tunisia, Egitto, India, Sry-Lanka, ecc.) e che sembrano mostrare una prevalenza in continuo aumento (24). Anch'esse sembrano indipendenti dai tradizionali fattori di rischio (ipertensione, diabete mellito, glomerulonefriti) e colpiscono soggetti che lavorano all'aperto in alcune delle zone più calde della terra o comunque in ambienti molto caldi (miniere). Nonostante l'agente eziologico sia ancora ignoto, è verosimile che l'ambiente caldo agisca almeno da fattore predisponente. Per questo, oggi, tra i fattori di rischio "non tradizionali" di MRC devono essere annoverate le alterazioni climatiche.

$E$ questo è un problema. Perché, indipendentemente dall'annosa questione sulla responsabilità o meno da attribuire all'uomo, è innegabile che siamo in un'era di progressivo riscaldamento globale e che la temperatura media del nostro pianeta sia aumentata più nelle ultime decadi che nei 1.400 anni precedenti. Da qui l'importanza di cercare di modificare le strutture economiche in direzione di una ridotta produzione di gas serra e di arrestare i processi di deforestazione.

\section{Contaminazione da polveri}

L'inquinamento ambientale non si limita ai gas serra e al surriscaldamento globale. Elevati livelli di particolato fine (con diametro <2,5 micron, PM2.5) si associano a un aumentato rischio di malattie cardiovascolari e di morte e ad altre conseguenza dannose per la salute. Nella zona degli Appalachi, un'alta mortalità per malattie renali nei minatori è stata attribuita ad un'elevata esposizione a materiale particolato (25). Altri Autori hanno descritto una relazione lineare fra esposizione a PM2.5 e incidenza di MRC, progressione della malattia renale e rischio di insufficienza renale cronica terminale $(26,27)$. Nel 2016, l'incidenza del carico globale di MRC attribuibile a PM2.5 in tutto il mondo è stata stimata pari a 6.950.514 casi, con una prevalenza di 94,29/100.000 abitanti. Anche in questo caso, contrariamente a quanto potrebbe sembrare intuibile, i Paesi a rischio più elevato si sono rivelati quelli a reddito basso e medio-basso. In particolare, il contributo della contaminazione aerea alla mortalità per MRC (e, più in generale, per malattie non infettive) sembra più pronunciato in quei Paesi che hanno avviato un processo di transizione epidemiologica (fase di sviluppo caratterizzata da un improvviso e significativo aumento della popolazione causato dal miglioramento delle condizioni nutritive e della sanità pubblica, seguito da un nuovo livellamento della popolazione per successivo declino del tasso di fertilità) e in fase di rapida industrializzazione (28).

\section{Le possibili strategie per un'ottimale prevenzione e gestione della MRC (e non solo)}

Sulla base di quanto detto finora, e in particolare a proposito dei molteplici e differenti fattori di rischio, è intuibile che una gestione ottimale della MRC debba prevedere strategie estremamente diversificate che mirino alla sua prevenzione secondaria e primaria ma anche (e soprattutto) "primordiale". Alcuni Autori (29) hanno presentato un ambizioso e complesso schema (Fig. 8) che cerca di inglobare tutti i possibili trattamenti dei vari tipi di prevenzione a seconda delle diverse età della vita. Tuttavia, in sintesi, mentre la prevenzione secondaria prevede terapie più strettamente mediche (come la diagnosi precoce della MRC e il trattamento del diabete mellito e dell'ipertensione arteriosa), nella prevenzione 


\begin{tabular}{|c|c|c|c|c|c|}
\hline PREVENZIONE & FETO/NEONATO & BAMBINO & ADOLESCENTE & ADULTO & ANZIANO \\
\hline PRIMORDIALE & $\begin{array}{ll}\text { - } & \text { SDGs } \\
\text { - } & \text { Salute dei genitori } \\
& \text { Gravidanza regolare }\end{array}$ & $\begin{array}{ll}\text { - } & \text { SDGs } \\
\text { - } & \text { Educare le bambine } \\
\text { - } & \text { Misure di salute pubblica }\end{array}$ & $\begin{array}{ll}\text { - } & \text { SDGs } \\
\text { - } & \text { Educare le bambine } \\
\text { - } & \text { Misure di salute pubblica }\end{array}$ & $\begin{array}{ll}\text { - } & \text { SDGs } \\
\text { - } & \text { Uguaglianza di genere } \\
\text { - } & \text { Misure di salute pubblica }\end{array}$ & $\begin{array}{ll}\text { - } & \text { SDGs } \\
\text { - } & \text { Invecchiamento "sano" } \\
\text { - Misure di salute pubblica }\end{array}$ \\
\hline PRIMARIA & $\begin{array}{ll}\text { - } & \text { Copertura economica } \\
\text { sanitaria universale } \\
\text { - } & \text { Salute del feto } \\
\text { - Stile di vita salutare ed } \\
\text { educazione madre e } \\
\text { bambino } \\
\text { - Profilassi pre- } \\
\text { eclampsia } \\
\text { Identificare madri ad } \\
\text { alto rischio di MRC, } \\
\text { MCV e DM } \\
\text { Evitare IRA }\end{array}$ & $\begin{array}{ll}\text { - } & \text { Educare a stile di vita } \\
\text { - } & \text { salutare } \\
\text { - } & \text { Alimentazione sana } \\
& \text { Copertura economica } \\
\text { - } & \text { sanitaria universale } \\
\text { - } & \text { Erevenzione obesità } \\
& \text { Evitare IRA }\end{array}$ & $\begin{array}{ll}\text { - } & \text { Educare a stile di vita } \\
& \text { salutare } \\
\text { - } & \text { Vita sessuale sana } \\
\text { - } & \text { Pianificazione familiare } \\
\text { - } & \text { Profilassi pre-eclampsia } \\
& \text { Evitare sostanze } \\
\text { - } & \text { Evirotossiche } \\
\text { - } & \text { Evitare fumo, alcol } \\
\text { - } & \text { Copertura economica } \\
& \text { sanitaria universale }\end{array}$ & $\begin{array}{ll}\text { - } & \text { Educare a stile di vita } \\
\text { - } & \text { salutare } \\
\text { - } & \text { Evitarassi pre-eclampsia } \\
& \text { nefrotossiche } \\
\text { - } & \text { Evitare fumo, alcol } \\
\text { - } & \text { Controllo peso } \\
\text { - } & \text { Evitare IRA } \\
\text { - } & \text { Screening gruppi ad alto } \\
\text { - } & \text { rischio } \\
& \text { Trattare ipertensione, DM } \\
\text { - } & \text { Copertinfezioni } \\
& \text { sanitaria universale }\end{array}$ & $\begin{array}{ll}\text { - } & \text { Educare a stile di vita } \\
\text { - } & \text { salutare } \\
& \text { Evitare sostanze } \\
\text { nefrotossiche } \\
\text { - } & \text { Evitare fumo, alcol } \\
\text { - } & \text { Copertura economica } \\
\text { - } & \text { Sanitaria universale } \\
\text { - Screening gruppi ad alto } & \text { rischio } \\
\text { - Trattare ipertensione, } \\
\text { DM ed infezioni } \\
\text { - Controllo peso } \\
\text { - Evitare IRA }\end{array}$ \\
\hline SECONDARIA & $\begin{array}{l}\text { Aggiustare dosi } \\
\text { farmaci in TI neonatale } \\
\text { Trattare uropatia } \\
\text { ostruttiva }\end{array}$ & $\begin{array}{l}\text { Follow-up bamblni nati } \\
\text { pretermine, con basso } \\
\text { peso o piccoli per età } \\
\text { gestazionale }\end{array}$ & $\begin{array}{l}\text { - Trattare ipertensione e } \\
\text { DM } \\
\text { - Evitare sovrappeso }\end{array}$ & $\begin{array}{l}\text { Precoci diagnosi e } \\
\text { trattamento MRC } \\
\text { Evitare IRA }\end{array}$ & $\begin{array}{l}\text { Precoci diagnosi e } \\
\text { trattamento MRC } \\
\text { Evitare IRA }\end{array}$ \\
\hline
\end{tabular}

Fig. 8 - Strategie per la prevenzione della MRC lungo il corso della vita. Da (29) modificato.

SDG (Sustainable Development Goals): Obiettivi di Sviluppo Sostenibile; MRC: Malattia Renale Cronica; MCV: Malattie CardioVascolari; DM: Diabete Mellito; IRA: Insufficienza Renale Acuta; TI: Terapia Intensiva.

primaria acquistano progressiva importanza strumenti di tipo sociale, mirati a facilitare uno stile di vita più sano (per esempio, evitare sovrappeso, fumo e alcol). E quando l'attenzione si sposta verso l'obiettivo ideale, quella prevenzione "primordiale" che dovrebbe impedire la comparsa di MRC (e dei suoi fattori di rischio) addirittura nelle generazioni future, allora i mezzi da utilizzare diventano "principalmente" di tipo politico-sociale e, in particolare, in ogni età della vita contemplano il raggiungimento degli SDG (Sustainable Development Goals). Cosa si intende con questo termine?

Partendo dalla convinzione che la maggior parte della mortalità sul nostro pianeta sia attribuibile alle cosiddette epidemie non trasmissibili e che una significativa percentuale di queste morti possa essere evitata attraverso l'eliminazione di fattori di rischio comuni (come fumo, alcol, errori dietetici, sedentarietà), nel 2015 l'Assemblea delle Nazioni Unite ha individuato 17 Obiettivi di Sviluppo Sostenibile (SDG) (Fig. 9) che, se ottenuti, dovrebbero avere un impatto positivo sulla salute attraverso politiche finalizzate con azioni sull'ambiente, sulle istituzioni e sulla società. A prima vista, questi obiettivi potrebbero essere considerati troppo ambiziosi e utopistici, ma forse qualcosa si può fare. Nel 2017 per la prima volta l'OMS ha effettuato un calcolo del costo finanziario necessario per affrontare le malattie coniche non trasmissibili nei Paesi a basso e medio-basso reddito e lo ha tradotto in termini di salute e ritorno economico (30). Ebbene, elaborando una strategia mirata a implementare un gruppo di interventi considerati efficaci e a basso costo (Fig. 10), spendere un dollaro in più ogni anno per ogni individuo porterebbe entro il 2030 quegli stessi Paesi a diminuire la mortalità prematura del $15 \%$, a prevenire complessivamente
17.000.000 di attacchi ischemici cardiaci e ictus e a evitare complessivamente 8.200 .000 morti. II fatto sorprendente è che tali benefici sulla salute si accompagnerebbero anche ad un guadagno netto di 6 dollari per ogni dollaro speso in più, per un guadagno complessivo di 350 bilioni di dollari!

Un'altra conferma del fatto che esista ancora spazio per migliorare la gestione della MRC arriva dalla cosiddetta "analisi di frontiera", che confronta il carico di MRC tra vari Paesi aggiustato per l'ISD di ciascun Paese. In altri termini, essa offre una valutazione quantitativa della potenziale diminuzione del carico di MRC che potrebbe essere ottenuta in un determinato Paese sulla base del suo sviluppo sociale ed economico. Questo tipo di analisi mostra che nel mondo molti Paesi sarebbero in grado, in base alle proprie risorse, di migliorare effettivamente la gestione/prevenzione della MRC (2).

In conclusione, è possibile una gestione ideale della pandemia da MRC? Ebbene, forse sì. Ma la soluzione è difficile perché le strategie adottate dovrebbero essere integrate a comprendere più in generale la prevenzione delle malattie epidemiche non trasmissibili (in primis, malattie cardiovascolari, diabete e obesità), dovrebbero prevedere molteplici punti d'azione e, infine, dovrebbero essere sostenute da regolamenti e leggi. Tanto per fare alcuni esempi, una gestione ideale dovrebbe implementare a livello pubblico un'alimentazione più sana (ad esempio con incentivi economici per diminuire i prezzi dei cibi più salutari e viceversa con una tassazione maggiore di quelli più nocivi), una minore sedentarietà (ad esempio, favorendo l'educazione fisica nelle scuole e la costruzione di aree pubbliche destinate all'attività motoria) e una diminuzione dell'inquinamento in tutte le sue forme (mediante politiche ambientali adeguate). 


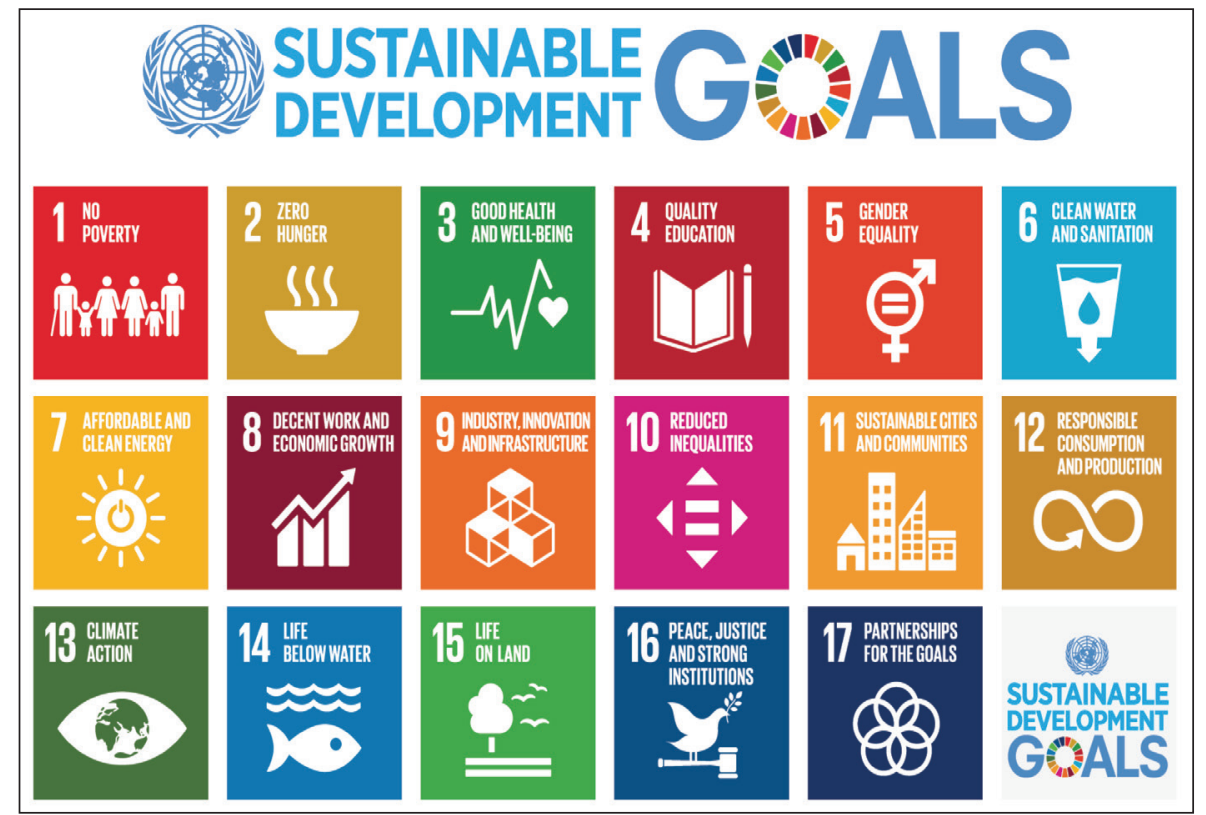

Fig. 9 - Obiettivi per uno sviluppo sostenibile indicati dall'Assemblea delle Nazioni Unite nel 2015.

1) Eliminare la povertà dovunque e in tutte le sue forme. 2) Eliminare la fame, ottenere cibi sicuri e un'alimentazione più sana e promuovere un'agricoltura sostenibile. 3) Assicurare vite sane e promuovere benessere psicofisico a tutte le età. 4) Assicurare una qualità di educazione inclusiva ed equa per tutti e promuovere l'apprendimento in tutte le età della vita. 5) Ottenere I'uguaglianza di genere e dare a ragazze e donne adulte le stesse possibilità del sesso maschile. 6) Assicurare a tutti l'accesso all'acqua e a un'igiene adeguata. 7) Assicurare a tutti la disponibilità di un'energia moderna, affidabile e sostenibile. 8) Promuovere uno sviluppo economico inclusivo e sostenibile e un lavoro decente per tutti. 9) Costruire infrastrutture resilienti, capaci di tollerare eventuali perturbazioni del sistema mantenendo la loro integrità strutturale e funzionale e promuovere un'industrializzazione e un'innovazione sostenibili. 10) Ridurre le ineguaglianze tra ed entro i Paesi. 11) Rendere le città inclusive, sicure, resilienti e sostenibili. 12) Assicurare un consumo e una produzione sostenibili. 13) Adottare misure urgenti per combattere i cambiamenti climatici e il loro impatto sull'ambiente. 14) Conservare e utilizzare in modo sostenibile oceani, mari e risorse marine. 15) Gestire le foreste in modo sostenibile, combattere la desertificazione, arrestare e invertire la degradazione del suolo e arrestare la perdita di biodiversità. 16) Promuovere pace, giustizia e società inclusive. 17) Rivitalizzare una collaborazione globale per uno sviluppo sostenibile.
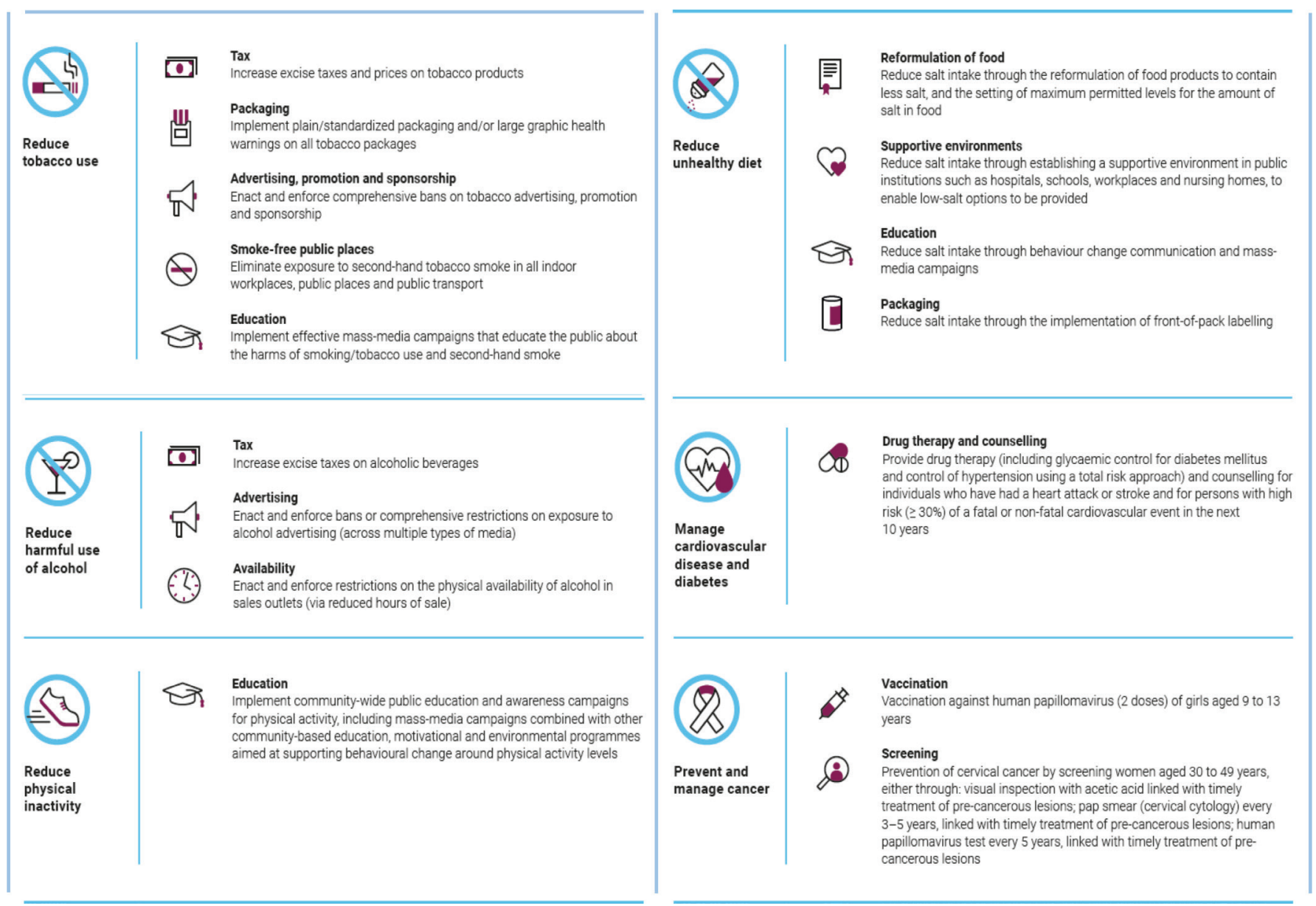

Fig. 10 - Interventi efficaci e a basso costo indicati dall'OMS nel 2017 per combattere le malattie croniche non trasmissibili nei Paesi a basso e medio-basso reddito (30). 


\section{Disclosures}

Conflict of interest: The author declares no conflict of interest. Financial support: This research received no specific grant from any funding agency in the public, commercial, or not-for-profit sectors.

\section{Bibliografia}

1. Annual Data Report//Chronic Kidney Disease//1: CKD in the General Population. Online (data di accesso ottobre 2021)

2. Xie Y, Bowe B, Mokdad AH, et al. Analysis of the Global Burden of Disease study highlights the global, regional, and national trends of chronic kidney disease epidemiology from 1990 to 2016. Kidney Int. 2018;94(3):567-581. CrossRef PubMed

3. Komenda P, Ferguson TW, Macdonald K, et al. Cost-effectiveness of primary screening for CKD: a systematic review. Am J Kidney Dis. 2014;63(5):789-797. CrossRef PubMed

4. Ministero della Salute. Documento di indirizzo per la malattia renale cronica. Online (data di accesso ottobre 2021)

5. Vassalotti JA, Centor R, Turner BJ, Greer RC, Choi M, Sequist TD; National Kidney Foundation Kidney Disease Outcomes Quality Initiative. Practical Approach to Detection and Management of Chronic Kidney Disease for the Primary Care Clinician. Am J Med. 2016;129(2):153-162.e7. CrossRef PubMed

6. International Diabetes Federation. Online (data di accesso ottobre 2021)

7. Mills KT, Stefanescu A, He J. The global epidemiology of hypertension. Nat Rev Nephrol. 2020;16(4):223-237. Online CrossRef

8. Bello AK, Levin A, Manns BJ, et al; Kidney Health for Life Initiative. Effective CKD care in European countries: challenges and opportunities for health policy. Am J Kidney Dis. 2015;65(1): 15-25. CrossRef PubMed

9. Bello AK, Levin A, Tonelli $M$, et al. Assessment of Global Kidney Health Care Status. JAMA. 2017;317(18):1864-1881. CrossRef PubMed

10. Bello AK, Alrukhaimi M, Ashuntantang GE, et al. Global overview of health systems oversight and financing for kidney care. Kidney Int Suppl (2011). 2018;8(2):41-51. CrossRef PubMed

11. Liyanage $\mathrm{T}$, Ninomiya $\mathrm{T}$, Jha $\mathrm{V}$, et al. Worldwide access to treatment for end-stage kidney disease: a systematic review. Lancet. 2015;385(9981):1975-1982. CrossRef PubMed

12. Haroun MK, Jaar BG, Hoffman SC, Comstock GW, Klag MJ, Coresh J. Risk factors for chronic kidney disease: a prospective study of 23,534 men and women in Washington County, Maryland. J Am Soc Nephrol. 2003;14(11):2934-2941. CrossRef PubMed

13. Yamagata K, Ishida K, Sairenchi T, et al. Risk factors for chronic kidney disease in a community-based population: a 10-year followup study. Kidney Int. 2007;71(2):159-166. CrossRef PubMed

14. Hallan S, de Mutsert R, Carlsen S, Dekker FW, Aasarød K, Holmen J. Obesity, smoking, and physical inactivity as risk factors for CKD: are men more vulnerable? Am J Kidney Dis. 2006;47(3):396-405. CrossRef PubMed

15. Merkin SS, Diez Roux AV, Coresh J, Fried LF, Jackson SA, Powe NR. Individual and neighborhood socioeconomic status and progressive chronic kidney disease in an elderly population: The Cardiovascular Health Study. Soc Sci Med. 2007;65(4): 809-821. CrossRef PubMed

16. Rose $D$, Richards R. Food store access and household fruit and vegetable use among participants in the US Food Stamp Program. Public Health Nutr. 2004;7(8):1081-1088. CrossRef PubMed

17. Dutko P, Ver Ploeg M, Farrigan T. Characteristics and influential factors of food deserts. [Washington, D.C.] US Dept of Agriculture, Economic Research Service; 2012.

18. Cameron AJ, Thornton LE, McNaughton SA, Crawford D. Variation in supermarket exposure to energy-dense snack foods by socio-economic position. Public Health Nutr. 2013;16(7): 1178-1185. CrossRef PubMed

19. Franco M, Diez Roux AV, Glass TA, Caballero B, Brancati FL. Neighborhood characteristics and availability of healthy foods in Baltimore. Am J Prev Med. 2008;35(6):561-567. CrossRef PubMed

20. Gutiérrez OM. Contextual poverty, nutrition, and chronic kidney disease. Adv Chronic Kidney Dis. 2015;22(1):31-38. CrossRef PubMed

21. Ruggajo P, Skrunes R, Svarstad E, Skjærven R, Reisæther AV, Vikse BE. Familial Factors, Low Birth Weight, and Development of ESRD: A Nationwide Registry Study. Am J Kidney Dis. 2016;67(4):601-608. CrossRef PubMed

22. Blencowe $\mathrm{H}$, Krasevec J, de Onis $\mathrm{M}$, et al. National, regional, and worldwide estimates of low birthweight in 2015, with trends from 2000: a systematic analysis. Lancet Glob Health. 2019;7(7):e849-e860. CrossRef PubMed

23. Hsu CW, Yamamoto KT, Henry RK, De Roos AJ, Flynn JT. Prenatal risk factors for childhood CKD. J Am Soc Nephrol. 2014;25(9):2105-2111. CrossRef PubMed

24. Johnson RJ, Wesseling C, Newman LS. Chronic Kidney Disease of Unknown Cause in Agricultural Communities. N Engl J Med. 2019;380(19):1843-1852. CrossRef PubMed

25. Hendryx M. Mortality from heart, respiratory, and kidney disease in coal mining areas of Appalachia. Int Arch Occup Environ Health. 2009;82(2):243-249. CrossRef PubMed

26. Mehta AJ, Zanobetti A, Bind MA, et al. Long-term exposure to ambient fine particulate matter and renal function in older men: The Veterans Administration Normative Aging study. Environ Health Perspect. 2016;124(9):1353-1360. CrossRef PubMed

27. Bowe B, Xie Y, Li T, Yan Y, Xian H, Al-Aly Z. Particulate Matter Air Pollution and the Risk of Incident CKD and Progression to ESRD. J Am Soc Nephrol. 2018;29(1):218-230. CrossRef PubMed

28. Bowe B, Xie Y, Li T, Yan Y, Xian H, Al-Aly Z. Estimates of the 2016 global burden of kidney disease attributable to ambient fine particulate matter air pollution. BMJ Open. 2019;9(5):e022450. CrossRef PubMed

29. Luyckx VA, Cherney DZI, Bello AK. Preventing CKD in Developed Countries. Kidney Int Rep. 2019;5(3):263-277. CrossRef PubMed

30. WHO. Institutional Repository for Information Sharing. Saving lives, spending less: a strategic response to noncommunicable diseases. Online (data di accesso ottobre 2021) 